\title{
Prenatal and childhood Mediterranean diet and the development of asthma and allergies in children
}

\author{
Leda Chatzi ${ }^{1, *}$ and Manolis Kogevinas ${ }^{1,2,3,4}$ \\ 'Department of Social Medicine, Faculty of Medicine, University of Crete, PO Box 2208, Heraklion 71003, \\ Crete, Greece: ${ }^{2}$ Centre for Research in Environmental Epidemiology (CREAL), Barcelona, Spain: ${ }^{3}$ Municipal \\ Institute of Medical Research (IMIM), Barcelona, Spain: ${ }^{4} \mathrm{CIBER}$, Epidemiologia y Salud Publica, \\ Barcelona, Spain
}

Submitted November 2008: Accepted April 2009

\begin{abstract}
Objective: To discuss current evidence about the relation between prenatal and childhood Mediterranean diet, and the development of asthma and allergies in children.

Design: Review of the literature.

Setting and results: Four recent studies conducted in Mediterranean countries (Spain, Greece) and one conducted in Mexico evaluated the association between childhood Mediterranean diet and asthma outcomes in children. All of the studies reported beneficial associations between a high level of adherence to the Mediterranean diet during childhood and symptoms of asthma or allergic rhinitis. Individual foods or food groups contributing to the protective effect of Mediterranean diet included fish, fruits, vegetables, legumes, nuts and cereals, while detrimental components included red meat, margarine and junk food intake.

Two studies focused on prenatal Mediterranean diet: the first is a birth cohort in Spain that showed a protective effect of a high adherence to the Mediterranean diet during pregnancy on persistent wheeze, atopic wheeze and atopy at the age of 6.5 years; while the second is a cross-sectional study in Mexico, collecting information more than 6 years after pregnancy, that showed no associations between maternal Mediterranean diet during pregnancy and allergic symptoms in childhood except for current sneezing.

Conclusions: Findings from recent studies suggest that a high level of adherence to the Mediterranean diet early in life protects against the development of asthma and atopy in children. Further studies are needed to better understand the mechanisms of this protective effect, to evaluate the most relevant window of exposure, and to address specific components of diet in relation to disease.
\end{abstract}

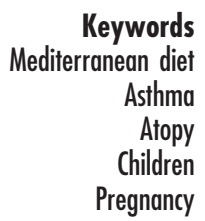

The prevalence of asthma and allergic diseases has increased dramatically over the past few decades with the highest incidence occurring in children ${ }^{(1)}$. One of the environmental changes that could have contributed to the recent increase in atopic diseases is diet. The modern diet is dominated by food that has been processed, modified, stored and transported great distances. This is in contrast to the traditional diet, which comprised food that was produced and marketed locally and was eaten shortly after harvesting ${ }^{(2)}$.

The traditional Mediterranean diet refers to a dietary pattern in the Mediterranean olive grove areas at the beginning of the $1960 \mathrm{~s}^{(3)}$. In general, the Mediterranean diet is characterised by elevated intake of plant foods such as fruits and vegetables, bread and cereals (primarily whole grain), legumes and nuts. Low to moderate amounts of dairy products and eggs, and only little amounts of red meat are included in the diet. This dietary pattern is low in saturated fatty acids, rich in carbohydrates, fibre, and antioxidants, and has a high content of monounsaturated fatty acids and $n-3$ PUFA, which are primarily derived from olive oil and fish intake ${ }^{(4)}$.

\section{Dietary hypotheses}

Two research hypotheses have been proposed while trying to explain the associations between diet and asthma. The first is the antioxidant hypothesis, proposed for the first time by Seaton et al. in $1994^{(5)}$. They suggested that a westernised diet, which was becoming progressively more deficient in antioxidants, increased the susceptibility of the population to allergens. Suboptimal dietary intake of antioxidant vitamins, especially vitamins $\mathrm{A}, \mathrm{C}$ and $\mathrm{E}$ and the carotenoids, as well as other 
antioxidants such as selenium and flavonoids may have an adverse effect on the modulation of oxidative lung stimuli; on the contrary, higher intakes may have beneficial associations with asthma, wheezing symptoms and ventilatory function $^{(6-8)}$. Moreover, the growth of airways during pregnancy or childhood may be vulnerable to oxidative exposures, while suboptimal antioxidant status during this critical period might result in oxidative airway damage, reductions in airway compliance or both ${ }^{(7)}$. In contrast to the data above on antioxidants, two recent birth cohorts reported that a higher intake of vitamin $\mathrm{C}$ and citrus fruits in pregnancy was associated with an increased risk of early eczema and allergic sensitisation, respectively ${ }^{(9,10)}$.

According to the lipid hypothesis, Black and Sharpe have proposed in 1997 that the increase in the prevalence of atopic diseases, especially in industrialised countries, has been preceded by a fall in the consumption of oily fish (containing long-chain $n-3$ PUFA) and an increase in the intake of fats containing $n$-6 PUFA (margarine and vegetable oils) resulting in an increase in the synthesis of prostaglandin E2 (PGE2) ${ }^{(11)}$. Through this mechanism, an increase in intake of $n-6$ PUFA could facilitate the development of asthma and allergy, because PGE2 suppresses differentiation into helper-inducer T-lymphocytes (Th1) cells, and promotes the Th2-cell phenotype by increasing class switching to $\operatorname{IgE}^{(2)}$.

\section{Maternal diet during pregnancy}

Epidemiological and immunological studies suggest that dietary modification or supplementation in the foetal life could reduce the development of atopic diseases, while foetal undernutrition could detrimentally affect the "programming' of the foetal lung and immune system ${ }^{(7,12,13)}$. Preterm birth has been consistently associated with a higher prevalence of wheezing illness and deficits in lung function measures in childhood ${ }^{(14,15)}$. Early life diet could modulate the likelihood of childhood asthma by affecting fetal airway development and/or influencing the initial early life interactions between allergens and the immune system by promoting Th-cell differentiation towards the Th2 cell type ${ }^{(16)}$. In animal models, vitamin $\mathrm{E}$, zinc and vitamin $\mathrm{D}$ have been shown to modify fetal lung development, and vitamin E, zinc, vitamin D and PUFA can modulate T-cell responses. In humans, birth cohort studies have reported associations between wheezing and eczema in early life and low prenatal status of selenium and iron ${ }^{(17)}$, low maternal intake of vitamin $\mathrm{E}^{(9,18,19)}$, vitamin $\mathrm{D}^{(20,21)}$, apple and fish ${ }^{(10,22,23)}$.

\section{Results}

\section{Mediterranean diet during pregnancy and the development of astbma and allergies in children}

Up to now, only two studies have reported associations between maternal Mediterranean diet during pregnancy and indicators of asthma and allergy in children (Table 1).
The first survey is a birth cohort study that started in 1997 in Menorca island, Spain ${ }^{(24)}$. Four hundred and sixty children were included in the analysis after 6.5 years of follow-up. Maternal dietary intake during pregnancy and children's dietary intake at an age of 6.5 years were assessed by FFQ. The degree of adherence to a traditional Mediterranean diet during pregnancy was based on the Mediterranean diet score, originally designed by Trichopoulou et al. ${ }^{(4)}$ with some adaptations. During follow-up, parents completed questionnaires on the child's respiratory and allergic symptoms.

One-third $(36 \cdot 1 \%)$ of the mothers had low quality of Mediterranean diet during pregnancy according to the Mediterranean diet score, while the rest had a high score. A high Mediterranean diet score during pregnancy (in two levels, using 'low' score as reference) was found to be protective for persistent wheeze (OR 0.22; 95\% CI $0 \cdot 08,0 \cdot 58)$, atopic wheeze (OR 0.30; $95 \%$ CI 0·10, 0.90) and atopy (OR $0.55 ; 95 \%$ CI $0.31,0.97)$ at age 6.5 years after adjusting for potential confounders. More specifically, consumption of vegetables more than eight times per week was inversely associated with persistent wheeze (OR 0.36; $95 \%$ CI 0.14, 0.92) and atopy (OR 0.40; 95\% CI $0 \cdot 22,0 \cdot 72)$. Fish intake more than two to three times per week and legumes intake more than once per week were inversely associated with persistent wheeze (OR 0.34; $95 \%$ CI $0 \cdot 13,0 \cdot 84$; OR 0.36; $95 \%$ CI $0 \cdot 13,1 \cdot 01$, respectively). In contrast, an increased intake of red meat (more than three to four times per week) showed a trend towards positive associations with persistent wheeze and atopic wheeze in offspring. When maternal and children Mediterranean diet index were simultaneously included in the multivariate models, results remained very similar, showing an independent beneficial effect of maternal diet during pregnancy on wheeze and atopy at 6.5 years of age.

The second study is a cross-sectional study conducted in 2004 using a random sample of 1476 children (6-7 years old) from the Mexicali region ${ }^{(25)}$. Parents were asked to complete the ISAAC (International Study of Asthma and Allergies in Childhood) questionnaire on respiratory and allergic symptoms and a 70-item FFQ asking both the diet of children in the last 12 months and the diet of their mothers during pregnancy. The degree of adherence to a traditional Mediterranean diet was based on the Mediterranean diet score, originally designed by Trichopoulou et $a l^{(4)}$, with some adaptations. Although the study has shown an inverse association between children's adherence to the Mediterranean diet, no associations were found using the mothers' pregnancy diet score, except for current sneezing (OR 0.71; $95 \%$ CI 0.53 , 0.97). The reported associations should be interpreted cautiously because of the reliability of maternal recall of diet 6-7 years previously. This could address significant recall bias in the study that limits in an important way the strength of its conclusions. 


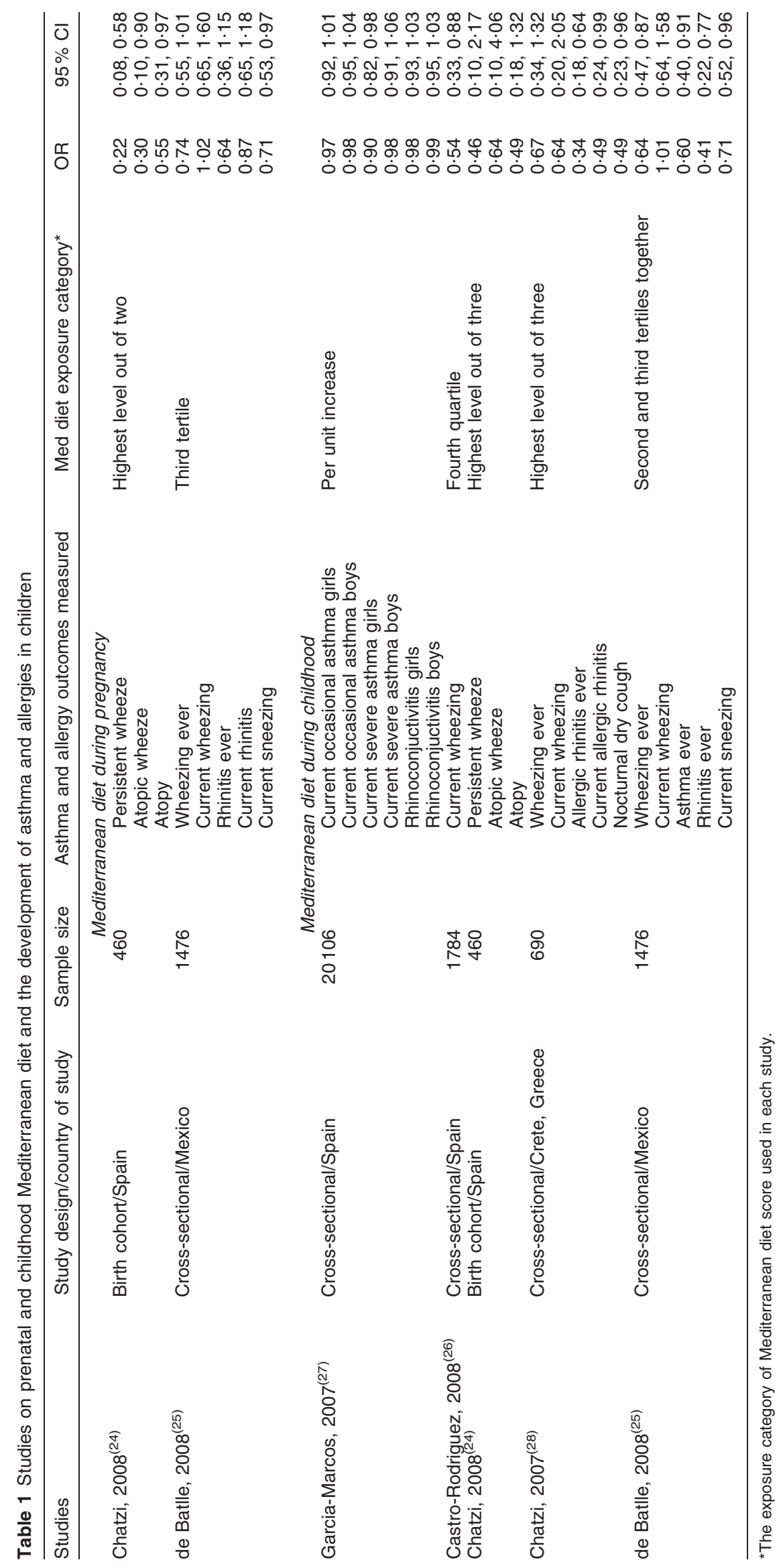




\section{Childbood Mediterranean diet and the} development of asthma and allergies in children

Four recent studies conducted in Mediterranean countries (Spain, Greece) $^{(24,26-28)}$ and one conducted in Mexico ${ }^{(25)}$ shed light on the association between Mediterranean diet and asthma outcomes in children (Table 1). The first survey in Spain was conducted in eight different Spanish cities among 20106 schoolchildren aged between 6 and 7 years $^{(27)}$. The parents were invited to complete the ISAAC III core and environmental questionnaires regarding asthma and rhinoconjuctivitis symptoms, dietary habits, weight and height, smoking habits of the mother, number of siblings and physical activity. The authors used a Mediterranean diet score that was based on a previously used score by Psaltopoulou et $a l .{ }^{(29)}$. The study has shown that an increase by one Mediterranean score unit had a small but protective effect on current severe asthma in girls (OR 0.90; 95\% CI $0 \cdot 82,0 \cdot 98)$, while obesity was the most significant risk factor (OR 2.35; 95\% CI 1.51, 3.64). Individually, a more frequent intake of seafood and cereals were protective factors for current severe asthma, while fast food was a risk factor. Seafood and fruit were protective factors also for rhinoconjunctivitis. A similar methodological study by CastroRodríguez et al., recruited 1784 preschool children in the province of Murcia in Spain ${ }^{(26)}$. The study has showed that being in the highest quartile of Mediterranean diet was a significant protective factor for current wheezing (OR 0.54; 95\% CI 0.3, 0.9), while, eczema, rhinoconjunctivitis, paternal asthma and acetaminophen consumption remained risk factors after controlling for several confounders.

The third study was a birth-cohort study conducted in Menorca island, Spain ${ }^{(24,30)}$. Parents completed a questionnaire on children's respiratory and allergic symptoms, and a 96-item FFQ. Children underwent skin prick tests with six common aeroallergens. The degree of adherence to a traditional Mediterranean diet was based on the KIDMED index, a Mediterranean diet quality index constructed to evaluate food habits in a population of Spanish children ${ }^{(31)}$. A low Mediterranean diet index was found for $9.3 \%$ of the children, $53.7 \%$ had intermediate values, and $37 \cdot 0 \%$ a high index. Multivariate logistic regression analysis showed negative associations, though not statistically significant, between a high level of adherence to the Mediterranean diet during childhood and persistent wheeze (OR 0•46; 95\% CI 0•10, 2.17), atopic wheeze (OR 0.64; 95\% CI 0.10, 4.06) and atopy (OR $0.49 ; 95 \%$ CI $0.18,1.32$ ) at age 6.5 years. More specifically, a high consumption $(>40 \mathrm{~g} / \mathrm{d})$ of fruity vegetables (tomatoes, eggplants, cucumber, green beans, zucchini) was found to have beneficial effect on current wheeze and atopic wheeze with a significant decreasing trend when intake was increased, while an inverse association was found between a high fish intake $(\geq 60 \mathrm{~g} / \mathrm{d})$ and atopy.

The study in Greece was conducted in 2001, in four rural areas of the Mediterranean island of Crete among
690 schoolchildren aged between 7 and 18 years ${ }^{(28)}$. The parents were invited to complete a questionnaire that included questions on the children's respiratory and allergic symptoms, family history of allergic diseases, birth order and sibling numbers, levels of parental education and occupation. A detailed 58-item FFQ was used to assess usual dietary intake in children. The degree of adherence to a traditional Mediterranean diet was based on the KIDMED index ${ }^{(31)}$. In all, $80 \%$ of children ate fresh fruit (and 68\% vegetables) at least twice a day. Daily consumption of grapes, oranges, apples and fresh tomatoes was found to have beneficial effect on wheezing symptoms. Daily intake of grapes was also inversely associated with current allergic rhinitis and current seasonal allergic rhinitis after adjusting for potential confounders. Consumption of nuts more than three times per week was inversely associated with wheezing (OR 0.54; 95\% CI 0.34, 0.86), whereas weekly intake of margarine appeared to be harmful on asthma (OR 2.19; $95 \%$ CI 1·01, $4 \cdot 82$ ) and allergic rhinitis symptoms (OR 1.99; $95 \%$ CI $1 \cdot 32,3 \cdot 00$ ). According to KIDMED index, $27 \cdot 9 \%$ of children had low quality of Mediterranean diet, $43 \cdot 8 \%$ had intermediate values and $28 \cdot 3 \%$ a high index. A high level of adherence to the Mediterranean diet was found to be protective for allergic rhinitis (OR 0.34; 95\% CI 0•18, 0.64), while a more modest protection was observed for wheezing (OR 0.64; $95 \%$ CI 0.20, 2.05) and atopy (OR 0.54; $95 \%$ CI $0 \cdot 21,1.99)$ though not statistically significant.

Finally, the study conducted in Mexico was a crosssectional study performed in 1476 children in Mexico ${ }^{(25)}$. Mothers were asked to complete a 70 -item FFQ asking both the diet of children in the last 12 months and their diet during pregnancy. The degree of adherence to a traditional Mediterranean diet was based on the Mediterranean diet score, originally designed by Trichopoulou et $a l^{(4)}$, with some adaptations. After adjusting for confounding factors, being in the two higher tertiles of Mediterranean diet score was inversely associated with asthma ever (OR 0•60; 95\% CI 0•40, 0•91), wheezing ever (OR 0.64; 95\% CI 0•47, 0•87), rhinitis ever (OR 0.41; 95\% CI $0 \cdot 22,0 \cdot 77$ ), current sneezing (OR $0.71 ; 95 \%$ CI 0.52 , $0 \cdot 96$ ) and current itchy-watery eyes (OR 0.63; 95\% CI $0 \cdot 42,0 \cdot 95)$. Children's junk food and fat consumption was positively associated, while cereals consumption was inversely associated with most of the outcomes.

\section{Summary, implications and the future}

Considerable advances in knowledge have been gained with studies focused on single nutrients or food items, however, these may fail to account for the interactions between nutrients, and they do not take into consideration that some nutrients are correlated between them ${ }^{(32)}$. Thus, interest has shifted to the study of food groups and, more recently, dietary patterns that represent a broader 
picture of food and nutrient consumption and may therefore be more predictive of disease risk. Dietary patterns such as Mediterranean diet account for cumulative and interactive effects among nutrients, reflect real-world-dietary preferences, and may be particularly suitable for analysis in asthma epidemiology where many dietary components could be related with the outcome of interest.

The results of the presented studies, indicating a protective effect of maternal (during pregnancy) and children's adherence to the Mediterranean diet on asthma and allergic disorders in childhood, reflect probably to a high exposure to several antioxidant compounds and long-chain $n$-3 PUFA and their adverse effect on the oxidative stress damage and the inflammation of lung tissues. However, these hypotheses have to be further elucidated, as recent studies have revealed the potential protective effect of non-antioxidants on airway and immune development, and some others have suggested that antioxidants may actually increase, instead of decrease, the risk of asthma and allergic disease $^{(33,34)}$. Thus, there is an increasing need to better understand the underlying mechanisms of the protective or the detrimental effect of certain foods or nutrients. Nutrients and biomarkers measured in maternal blood during pregnancy, umbilical cord blood or child blood may be useful to validate dietary intake more precisely. Moreover, experimental studies in animals could be a further step to confirm or refute causal links, before proceeding to intervention studies or randomised trial cohorts to investigate the impact of prenatal and childhood Mediterranean diet on the development of asthma and allergies in children.

The time window of exposure is becoming a key aspect in the study of diseases involving systems with a long developmental length such as the immunological and respiratory system ${ }^{(35)}$. It is possible that the immunomodulating benefits of antioxidant compounds may be greater during critical stages of early immune development before allergic response is established. Allergenspecific responses are already evident at birth and allergic disease is often manifest within the first month of life, suggesting that the processes that lead to allergic diseases can be initiated very early in immune development ${ }^{(13,23)}$. On the other hand, it is well established that during gestation, essential nutrients are transferred from the maternal to the foetal circulation across the placenta, and such transport mechanisms have been identified for antioxidants and long-chain PUFA, basic principles of the Mediterranean diet ${ }^{(36)}$.

The term 'Mediterranean diet' reflects the dietary patterns characteristic of several countries in the Mediterranean Basin during the early 1960s. Dietary scores like Mediterranean diet score or KIDMED index have been used widely to explore the multiple associations between Mediterranean diet and different health outcomes. Although these indexes are extremely useful tools to measure the degree of adherence to the Mediterranean diet, we cannot acknowledge some of their limitations such as the variability in choosing cut-off points in the score and the different distribution of selected food groups in different populations. Thus, a more precise and quantitative definition of the Mediterranean diet is required if the adherence to such a dietary pattern is intended to be more accurately measured ${ }^{(37)}$.

In conclusion, findings from the present review indicate that a high level of adherence to the Mediterranean diet early in life protects against asthma-like symptoms and atopy in childhood. Further studies are needed to better understand the mechanisms of this protective effect, to evaluate the most relevant window of exposure, and to address specific components of diet in relation to disease.

\section{Acknowledgements}

All authors had a substantial contribution to the study, and they have all personally reviewed and approved the submitted manuscript. Specifically, L.C. conceived the study and wrote the paper and M.C. participated in the design and in drafting the paper. None of the authors have any commercial or financial involvements that might present an appearance of a conflict of interest in connection with the submitted article. Dr Chatzi was supported, in part, by the EU Integrated Project NewGeneris, 6th Framework Programme (Contract no. FOOD-CT2005-016320).

\section{References}

1. Pearce N, Ait-Khaled N, Beasley R, Mallol J, Keil U, Mitchell E \& Robertson C (2007) Worldwide trends in the prevalence of asthma symptoms: phase III of the International Study of Asthma and Allergies in Childhood (ISAAC). Thorax 62, 758-766.

2. Devereux G (2006) The increase in the prevalence of asthma and allergy: food for thought. Nature Rev $\mathbf{6}$, 869-874.

3. Trichopoulou A (2001) Mediterranean diet: the past and the present. Nutr Metab Cardiovasc Dis 11, 1-4.

4. Trichopoulou A, Costacou T, Bamia C \& Trichopoulos D (2003) Adherence to a Mediterranean diet and survival in a Greek population. N Engl J Med 348, 2599-2608.

5. Seaton A, Godden DJ \& Brown K (1994) Increase in asthma: a more toxic environment or a more susceptible population? Thorax 49, 171-174.

6. McKeever TM \& Britton J (2004) Diet and asthma. Am J Respir Crit Care Med 170, 725-729.

7. Devereux G \& Seaton A (2005) Diet as a risk factor for atopy and asthma. J Allergy Clin Immunol 115, 1109-1117; quiz 18.

8. Romieu I \& Trenga C (2001) Diet and obstructive lung diseases. Epidemiol Rev 23, 268-287.

9. Martindale S, McNeill G, Devereux G, Campbell D, Russell G \& Seaton A (2005) Antioxidant intake in pregnancy in relation to wheeze and eczema in the first two years of life. Am J Respir Crit Care Med 171, 121-128.

10. Sausenthaler S, Koletzko S, Schaaf B, Lehmann I, Borte M, Herbarth O, von Berg A, Wichmann HE \& Heinrich J (2007) Maternal diet during pregnancy in relation to eczema and 
allergic sensitization in the offspring at 2 y of age. Am J Clin Nutr 85, 530-537.

11. Black PN \& Sharpe S (1997) Dietary fat and asthma: is there a connection? Eur Respir J 10, 6-12.

12. Langley-Evans S (1997) Fetal programming of immune function and respiratory disease. Clin Exp Allergy 27, $1377-1379$.

13. Tricon S, Willers S, Smit HA et al. (2006) Nutrition and allergic disease. Clin Exp Allergy Rev 6, 117-188.

14. Caudri D, Wijga A, Gehring U, Smit HA, Brunekreef B, Kerkhof M, Hoekstra M, Gerritsen J \& de Jongste JC (2007) Respiratory symptoms in the first 7 years of life and birth weight at term: the PIAMA birth cohort. Am J Respir Crit Care Med 175, 1078-1085.

15. Gregory A, Doull I, Pearce N, Cheng S, Leadbitter P, Holgate S \& Beasley R (1999) The relationship between anthropometric measurements at birth: asthma and atopy in childhood. Clin Exp Allergy 29, 330-333.

16. Devereux G (2007) Early life events in asthma - diet. Pediatr Pulmonol 42, 663-673.

17. Shaheen SO, Newson RB, Henderson AJ, Emmett PM, Sherriff A \& Cooke M (2004) Umbilical cord trace elements and minerals and risk of early childhood wheezing and eczema. Eur Respir J 24, 292-297.

18. Litonjua AA, Rifas-Shiman SL, Ly NP, Tantisira KG, RichEdwards JW, Camargo CA Jr, Weiss ST, Gillman MW \& Gold DR (2006) Maternal antioxidant intake in pregnancy and wheezing illnesses in children at 2 y of age. Am J Clin Nutr 84, 903-911.

19. Devereux G, Turner SW, Craig LC, McNeill G, Martindale S, Harbour PJ, Helms PJ \& Seaton A (2006) Low maternal vitamin $\mathrm{E}$ intake during pregnancy is associated with asthma in 5-year-old children. Am J Respir Crit Care Med 174, 499-507.

20. Camargo CA Jr, Rifas-Shiman SL, Litonjua AA, Rich-Edwards JW, Weiss ST, Gold DR, Kleinman K \& Gillman MW (2007) Maternal intake of vitamin D during pregnancy and risk of recurrent wheeze in children at 3 y of age. Am J Clin Nutr 85, 788-795.

21. Devereux G, Litonjua AA, Turner SW, Craig LC, McNeill G, Martindale S, Helms PJ, Seaton A \& Weiss ST (2007) Maternal vitamin D intake during pregnancy and early childhood wheezing. Am J Clin Nutr 85, 853-859.

22. Willers SM, Devereux G, Craig LC, McNeill G, Wijga AH, Abou El-Magd W, Turner SW, Helms PJ \& Seaton A (2007) Maternal food consumption during pregnancy and asthma, respiratory and atopic symptoms in 5-year-old children. Thorax 62, 773-779.

23. Romieu I, Torrent M, García-Esteban R, Ferrer C, Ribas-Fito N, Anto JM \& Sunyer J (2007) Maternal fish intake during pregnancy and atopy and asthma in infancy. Clin Exp Allergy 37, 518-525.

24. Chatzi L, Torrent M, Romieu I, García-Esteban R, Ferrer C, Vioque J, Kogevinas M \& Sunyer J (2008) Mediterranean diet in pregnancy is protective for wheeze and atopy in childhood. Thorax 63, 507-513.

25. de Batlle J, Garcia-Aymerich J, Barraza-Villarreal A, Anto JM \& Romieu I (2008) Mediterranean diet is associated with reduced asthma and rhinitis in Mexican children. Allergy 63, 1310-1316

26. Castro-Rodriguez JA, García-Marcos L, Alfonseda Rojas JD, Valverde-Molina J \& Sanchez-Solis M (2008) Mediterranean diet as a protective factor for wheezing in preschool children. J Pediatr 152, 823-828, 8 e1-2.

27. García-Marcos L, Canflanca IM, Garrido JB et al. (2007) Relationship of asthma and rhinoconjunctivitis with obesity, exercise and Mediterranean diet in Spanish schoolchildren. Thorax 62, 503-508.

28. Chatzi L, Apostolaki G, Bibakis I, Skypala I, Bibaki-Liakou V, Tzanakis N, Kogevinas M \& Cullinan P (2007) Protective effect of fruits, vegetables and the Mediterranean diet on asthma and allergies among children in Crete. Thorax $\mathbf{6 2}$, 677-683.

29. Psaltopoulou T, Naska A, Orfanos P, Trichopoulos D, Mountokalakis T \& Trichopoulou A (2004) Olive oil, the Mediterranean diet, and arterial blood pressure: the Greek European Prospective Investigation into Cancer and Nutrition (EPIC) Study. Am J Clin Nutr 80, 1012-1018.

30. Chatzi L, Torrent M, Romieu I, Garcia-Esteban R, Ferrer C, Vioque J, Kogevinas M \& Sunyer J (2007) Diet, wheeze, and atopy in school children in Menorca, Spain. Pediatr Allergy Immunol 18, 480-485.

31. Serra-Majem L, Ribas L, Garcia A, Perez-Rodrigo C \& Aranceta J (2003) Nutrient adequacy and Mediterranean diet in Spanish school children and adolescents. Eur J Clin Nutr 57, Suppl. 1, S35-S39.

32. Jacobs DR Jr \& Steffen LM (2003) Nutrients, foods, and dietary patterns as exposures in research: a framework for food synergy. Am J Clin Nutr 78, 508S-513S.

33. Shaheen SO (2008) Prenatal nutrition and asthma: hope or hype? Thorax 63, 483-485.

34. Murr C, Schroecksnadel K, Winkler C, Ledochowski M \& Fuchs D (2005) Antioxidants may increase the probability of developing allergic diseases and asthma. Med Hypotheses 64, 973-977.

35. Sunyer J, Torrent M, Munoz-Ortiz L, Ribas-Fito N, Carrizo D, Grimalt J, Anto JM \& Cullinan P (2005) Prenatal dichlorodiphenyldichloroethylene (DDE) and asthma in children. Environ Health Perspect 113, 1787-1790.

36. Schenker S, Yang Y, Perez A, Acuff RV, Papas AM, Henderson G \& Lee MP (1998) Antioxidant transport by the human placenta. Clin Nutr 17, 159-167.

37. Bach A, Serra-Majem L, Carrasco JL, Roman B, Ngo J, Bertomeu I \& Obrador B (2006) The use of indexes evaluating the adherence to the Mediterranean diet in epidemiological studies: a review. Public Health Nutr 9, 132-146. 